\title{
Sensor Data Integration for Indoor Human Tracking
}

\author{
J. A. CORRALES*, F. A. CANDELAS and F. TORRES \\ Physics, Systems Engineering and Signal Theory Department, University of Alicante \\ Carretera San Vicente del Raspeig s/n, 03690 San Vicente del Raspeig, Spain \\ [jcorrales;Francisco.Candelas;Fernando.Torres]@ua.es
}

\begin{abstract}
A human tracking system based on the integration of the measurements from an inertial motion capture system and a UWB (Ultra-Wide Band) location system has been developed. On the one hand, the rotational measurements from the inertial system are used to track precisely all limbs of the body of the human. On the other hand, the translational measurements from both systems are combined by three different fusion algorithms (a Kalman filter, a particle filter and a combination of both) in order to obtain a precise global localization of the human in the environment. Several experiments have been performed to compare their accuracy and computational efficiency.
\end{abstract}

Keywords: Indoor location, Motion Capture, Human-Robot Interaction, Kalman Filter, Particle Filter.

\section{INTRODUCTION}

In industrial environments where humans and robots share the same workspace, a precise localization of each human operator is needed. This localization does not only avoid dangerous collisions between humans and robots but it also provides valuable context information for the task planners of the robots $[1,2]$. Thus, human-robot interaction tasks become more natural and unobtrusive because trajectories of the robots are dynamically changed depending on the human operators' movements.

However, global positioning of the human operator in the workspace is not sufficient for close collaborative tasks. In these tasks, a full-body movement registration system is needed in order to detect precisely possible collisions between each limb of the human operator and each link of the industrial robot. Motion capture systems satisfy this requirement because they are able to track precisely the movements of the main limbs of a human being. A motion capture system based on inertial sensors has been chosen for the current research because of the advantages of this sensor technology [3]: small size, self-containment and no line-of-sight restrictions. Although the relative joint rotation measurements obtained from the inertial sensors are very precise, the global position of 
the human estimated by this system accumulates an important error through time [4]. Thus, an additional location system based on UWB pulses has been included in order to obtain more precise measurements of the global position of the human operator in the industrial workplace.

Three novel fusion algorithms based on Bayesian filters have been developed in order to combine the position measurements of these two tracking systems. The first algorithm is based on a Kalman filter, the second one is based on a particle filter and the third one is based on a combination of a Kalman filter and a particle filter. These fusion algorithms modify the standard structure of the Bayesian filters in order to adjust them to the features of the measurements of the tracking systems. On one hand, position accuracy of the UWB location system is used to correct the error accumulation of the inertial motion capture system. On the other hand, the high sampling rate of the inertial motion capture system is used to continue the tracking process while the UWB system does not supply any measurement.

\section{PREVIOUS WORK ON SENSOR FUSION}

Inertial sensors obtain an estimation of the pose of the objects to which they are attached by integrating their measurements. However, this integration process implies the accumulation of a small drift which becomes significant after several integration steps [5]. Previous research corrects this integration drift by combining the measurements of the inertial sensors with the position measurements of other sensors (e.g. GPS, ultrasound, magnetic, cameras, UWB and Wi-Fi). Different adaptations of Bayesian filtering techniques [6] (especially, Kalman filters and particle filters) have been proposed in order to estimate probabilistically the system's pose from the noisy measurements of the sensors.

The generic Kalman filter [7] has been adapted in two different ways in order to incorporate measurements from different sensors: complementary Kalman filters and Kalman filters with multichannel correction steps. Tracking systems based on Complementary Kalman filters [8-10] receive as input the differences between the sensors combining the measurements into a common value which is applied to the filter. Thereby, they estimate sensor errors instead of direct measurements. The other type of Kalman filter adaptations are based on the modification of the correction step of the filter in order to incorporate several measurements. In particular, a measurement model for each sensor type is defined and applied independently in the correction step of the Kalman filter [11]. These correction channels can be weighted according to fuzzy variables which represent the reliability of each sensor [12]. Particle filters have also been modified in a similar way and different measurement models are defined for each sensor in hybrid tracking systems [13-15]. These measurement models are used to update the weights of the particles of these filters during the importance factor computation step.

The first two algorithms which are developed in this research are based on a Kalman filter and a particle filter, respectively. These algorithms improve the computational cost of the fusion approaches described above by reducing the execution frequency of their internal steps (prediction 
and correction). They perform only one of the steps of the filters depending on the received measurement: prediction step for inertial measurements and correction step for UWB measurements. In [16], a similar Kalman-based approach is presented for a human tracking system, which applies the prediction step of the filter to the measurements from inertial sensors and the correction step to the measurements from other additional sensors. Nevertheless, the algorithm in [16] executes both the prediction and correction steps of the filter at each iteration. This fact involves that the additional sensors, which are used to correct the inertial drift, must register measurements at a similar sampling rate. This limitation is overcome by the algorithms proposed in this paper because one of their main advantages is the fusion of measurements from tracking systems with different sampling rates. Finally, the third fusion algorithm described in this paper uses both filters (Kalman and particle) in order to combine their advantages and obtain more accurate position estimates with a smaller computational cost than the stand-alone particle filter.

\section{TRACKING SYSTEM HARDWARE COMPONENTS}

The human tracking system described in this paper is composed of two main devices: an inertial motion capture system (GypsyGyro-18 from Animazoo) and a UWB location system (from Ubisense).

The inertial motion capture system is composed of 18 small IMUs (Inertial Measurement Units) attached to a lycra suit which is worn by the human operator. Fig. 1.a shows this suit and the location of the IMUs over it. Each IMU measures the orientation (roll, pitch and yaw) of the operator's limb to which it is attached by combining the measurements of three MEMS (Micro-Electro-Mechanical Systems) gyroscopes, three accelerometers and three magnetometers. These measurements are represented in a 3D skeleton structure (see Fig. 1.b). This skeleton represents the human body as a hierarchical structure where the motion of each node is relative to the motion of its predecessor nodes. The root node of this hierarchy is the hips node and it represents the global position of the human in the environment. 


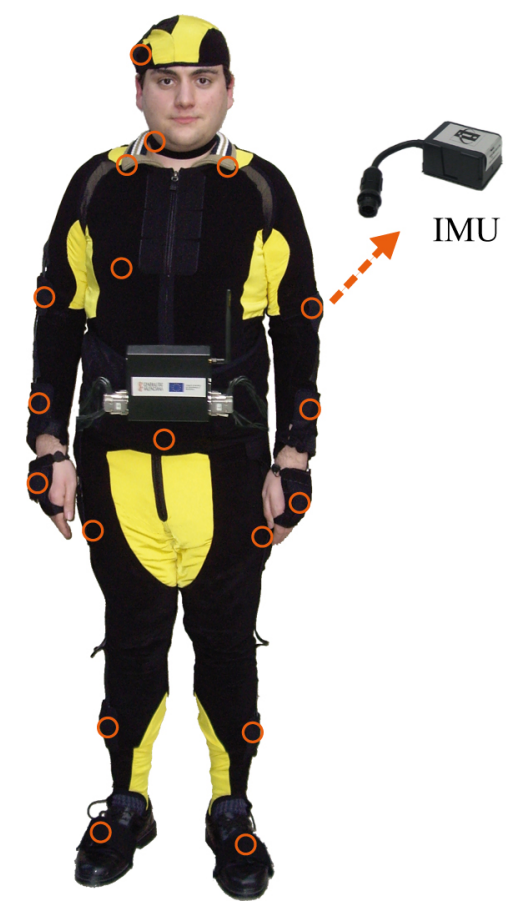

(a)

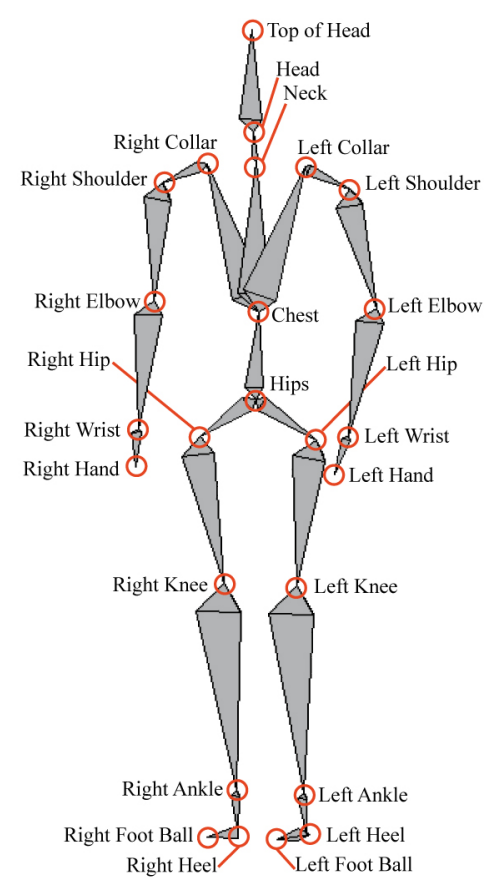

(b)

Figure 1: Inertial motion capture system: (a) motion capture suit composed by 18 IMUs; (b) skeleton structure where the measurements from the suit are applied.

The relative rotations of each node of the skeleton are very precise because they are directly obtained from the orientation measurements registered by the IMUs. However, the global translation of the hips node is estimated in the controller PC by a footstep extrapolation algorithm which accumulates important errors over time (see [4] for a detailed set of experiments which quantify these errors). Thus, an additional localization system which provides precise global translation measurements is needed. A wireless localization system based on Ultra-Wideband (UWB) pulses has been used in the work described in this paper. The human operator carries a small device (tag) which sends UWB pulses to four sensors which are situated at fixed positions on the localization area. A more precise estimate of the 3D global position of the human is obtained by applying TDOA (Time-Difference of Arrival) and AOA (Angle of Arrival) techniques to these UWB pulses.

\section{SENSOR FUSION ALGORITHMS}

\subsection{Sensor fusion principles}

The three Bayesian filter algorithms developed in this paper are based on the complementary features of the global translation measurements of both tracking systems. On one hand, the translation measurements of the inertial motion capture system accumulate substantial errors over time while the translation measurements of the UWB system are more precise. Thereby, the implemented fusion algorithms use the measurements of the UWB system as ground truth data for removing the accumulated errors of the inertial system. On the other hand, the UWB system 
registers measurements with a lower rate $(5-9 \mathrm{~Hz})$ than the inertial system $(30-120 \mathrm{~Hz})$. Thus, the implemented fusion algorithms use the measurements of the inertial system to estimate the position of the skeleton between each pair of UWB measurements. The resulting system from the fusion of both tracking systems will have a higher sampling rate and a better accuracy than each system separately.

The developed fusion algorithms adapt a Kalman filter, a particle filter and a combination of a Kalman filter and a particle filter in order to consider these complementary features of both tracking systems. These algorithms will be exposed in detail in subsequent subsections. Nevertheless, the first step to combine global position measurements of both tracking systems is to represent them in the same coordinate system. The transformation equations needed to do so are explained next.

\subsection{Coordinate transformation}

The position measurements from the UWB localization system are represented in the frame $U$ while the position measurements from the inertial motion capture system are represented in the frame $G$ (see Fig. 2). The frame $U$ is a static coordinate system which is established according to the fixed positions where the UWB sensors are installed. However, the frame $G$ is dynamic since its origin is established in the place where the user initializes the system. The $U$ frame of the UWB system has been selected as the reference coordinate system because it allows comparing easily the position of the human operator with the position of static objects (like machinery) in the environment.

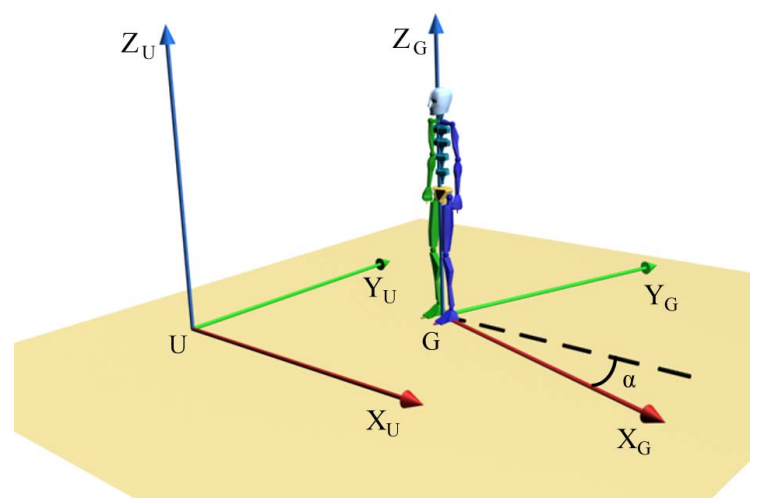

Figure 2. Coordinate systems of the UWB and the inertial motion capture system.

The XY planes of the $U$ and $G$ frames are parallel to the plane of the floor in the environment. Therefore, between them there is only a translation $\left(x_{G}^{U}, y_{G}^{U}, z_{G}^{U}\right)$ and a rotation about the $Z$ axis by an angle $\alpha$. Each position measurement $\left(x^{G}, y^{G}, z^{G}\right)$ of the inertial motion capture system can be represented in the UWB coordinate system $\left(x^{U}, y^{U}, z^{U}\right)$ by applying the following transformation matrix ${ }^{U} T_{G}$ : 


$$
\left[\begin{array}{c}
x^{U} \\
y^{U} \\
z^{U} \\
1
\end{array}\right]=\left[\begin{array}{cccc}
\cos (\alpha) & -\sin (\alpha) & 0 & x_{G}^{U} \\
\sin (\alpha) & \cos (\alpha) & 0 & y_{G}^{U} \\
0 & 0 & 1 & z_{G}^{U} \\
0 & 0 & 0 & 1
\end{array}\right] \cdot\left[\begin{array}{c}
x^{G} \\
y^{G} \\
z^{G} \\
1
\end{array}\right]
$$

The parameter $\alpha$ in this transformation matrix is a known constant value which represents the angle between the $Y$ axis of both frames. The three coordinates of the translation vector $\left(x_{G}^{U}, y_{G}^{U}, z_{G}^{U}\right)$ between frame $U$ and frame $G$ are calculated from (1) by substituting two corresponding measurements from both systems:

$$
\begin{gathered}
x_{G}^{U}=x^{U}-x^{G} \cos (\alpha)+y^{G} \sin (\alpha) \\
y_{G}^{U}=y^{U}-x^{G} \sin (\alpha)-y^{G} \cos (\alpha) \\
z_{G}^{U}=z^{U}-z^{G}
\end{gathered}
$$

After obtaining the parameters of the transformation matrix ${ }^{U} T_{G}$, all the translational measurements from the motion capture system will be transformed to the UWB frame by applying (1).

\subsection{Kalman filter fusion approach}

Kalman filters are the most widely used technique to implement Bayesian filters. The generic Kalman filter algorithm [7] has been modified in order to incorporate sensor measurements from the two tracking systems by considering their complementary features: high sampling rate of the inertial motion capture system and location precision of the UWB system.

The state $x_{t}$ which should be estimated by the Kalman filter developed for the work described in this paper is composed by the coordinates $p=(x, y, z)$ of the global position of the human operator in the environment (position of the hips node of the skeleton). The prediction step of this Kalman filter uses a state evolution model where the estimate of the state $\hat{x}_{t}$ is obtained from the current measurement of the inertial motion capture system $z_{\text {inertial }}(t)$ plus a Gaussian noise $w_{\text {inertial }}(t)$ :

$$
\hat{x}_{t}=z_{\text {inertial }}(t)+w_{\text {inertial }}(t)
$$

This model is implemented in the Kalman filter prediction step using the following two equations:

$$
\begin{gathered}
\hat{x}_{t}=A z_{\text {inertial }}(t) \\
P_{t}=A P_{t-1} A^{T}+Q
\end{gathered}
$$

The matrix A represents the state transition model of (5) and thus it is a $3 \times 3$ identity matrix (with ones on the main diagonal and zeros elsewhere) which uses the last measurement from the motion capture system as the current state estimate. $P_{t}$ is the error covariance matrix which represents the accuracy of the state estimate $\hat{x}_{t} . Q$ is the $3 \times 3$ covariance matrix of the error $w_{\text {inertial }}(t)$ of the inertial motion capture system. It is a diagonal matrix whose terms represent the 
variance of the position errors of the inertial system. The terms of this matrix are adjusted experimentally from a representative group of experiments so that the errors of the final state estimates are minimum.

The correction step of the filter is based on an observation model where the measurements from the UWB system $\hat{z}_{u w b}(t)$ are approximated by the last state estimate of the prediction step $\hat{x}_{t-1}$ plus a Gaussian noise $w_{u w b}(t)$ :

$$
\hat{z}_{u w b}(t)=\hat{x}_{t-1}+w_{u w b}(t)
$$

This observation model is implemented in the Kalman filter correction step using the following three equations:

$$
\begin{gathered}
K_{t}=P_{t-1} H^{T}\left(H P_{t-1} H^{T}+R\right) \\
\hat{x}_{t}=\hat{x}_{t-1}+K_{t}\left(z_{u w b}(t)-H \hat{x}_{t-1}\right) \\
P_{t}=\left(I-K_{t} H\right) P_{t-1}
\end{gathered}
$$

Position measurements from the UWB system are used as observations $z_{u w b}(t)$ in this step in order to correct the predicted position $\hat{x}_{t-1}$ calculated from the motion capture system. The matrix $H$ represents the measurement model of (8) and it is a $3 \times 3$ identity matrix (with ones on the main diagonal and zeros elsewhere) because UWB measurements have the same dimension as the state. $R$ is the $3 \times 3$ covariance matrix of the error $w_{u w b}(t)$ of the UWB system. It is a diagonal matrix whose terms represent the variance of the position errors of the UWB system. The terms of this matrix are adjusted experimentally from a representative group of experiments so that the errors of the final state estimates are minimum.

These prediction and correction steps of the Kalman filter are implemented by the algorithm shown in Table 1. The main advantage of this algorithm over previous sensor fusion techniques based on Kalman filtering is that only one step of the algorithm (prediction or correction) is executed each time a measurement is obtained. The prediction step ((6), (7)) is executed when a measurement from the inertial motion capture system is received (line 9 of Table 1 ) while the correction step ((9)(11)) is executed when UWB measurements are received (line 12 of Table 1). Thereby, the execution time of the algorithm is smaller than other fusion algorithms based on the Kalman filter ([8-12]) which complete both filter steps at each iteration.

This algorithm reduces the drawbacks of both tracking systems by combining their complementary features. On one hand, the error accumulated by the inertial motion capture system is removed after each correction step execution because the transformation matrix ${ }^{U} T_{G}$ is re-calculated (line 6 of Table 1) with the last state estimate obtained from a UWB measurement. This new matrix ${ }^{U} T_{G}$ is used in the following prediction step executions in order to represent the inertial system measurements in the UWB coordinate system (line 8 of Table 1) and thus the drift of previous inertial measurements is corrected. On the other hand, the low sampling rate of the UWB 
measurements does not reduce the latency of the system because the inertial system measurements registered between each pair of UWB measurements are used in the prediction step in order to obtain an estimate of the system's state (line 9 of Table 1). Thereby, this algorithm provides state estimates with a high sampling rate which is suitable to track quick human movements.

Table 1: Fusion algorithm based on Kalman filter.

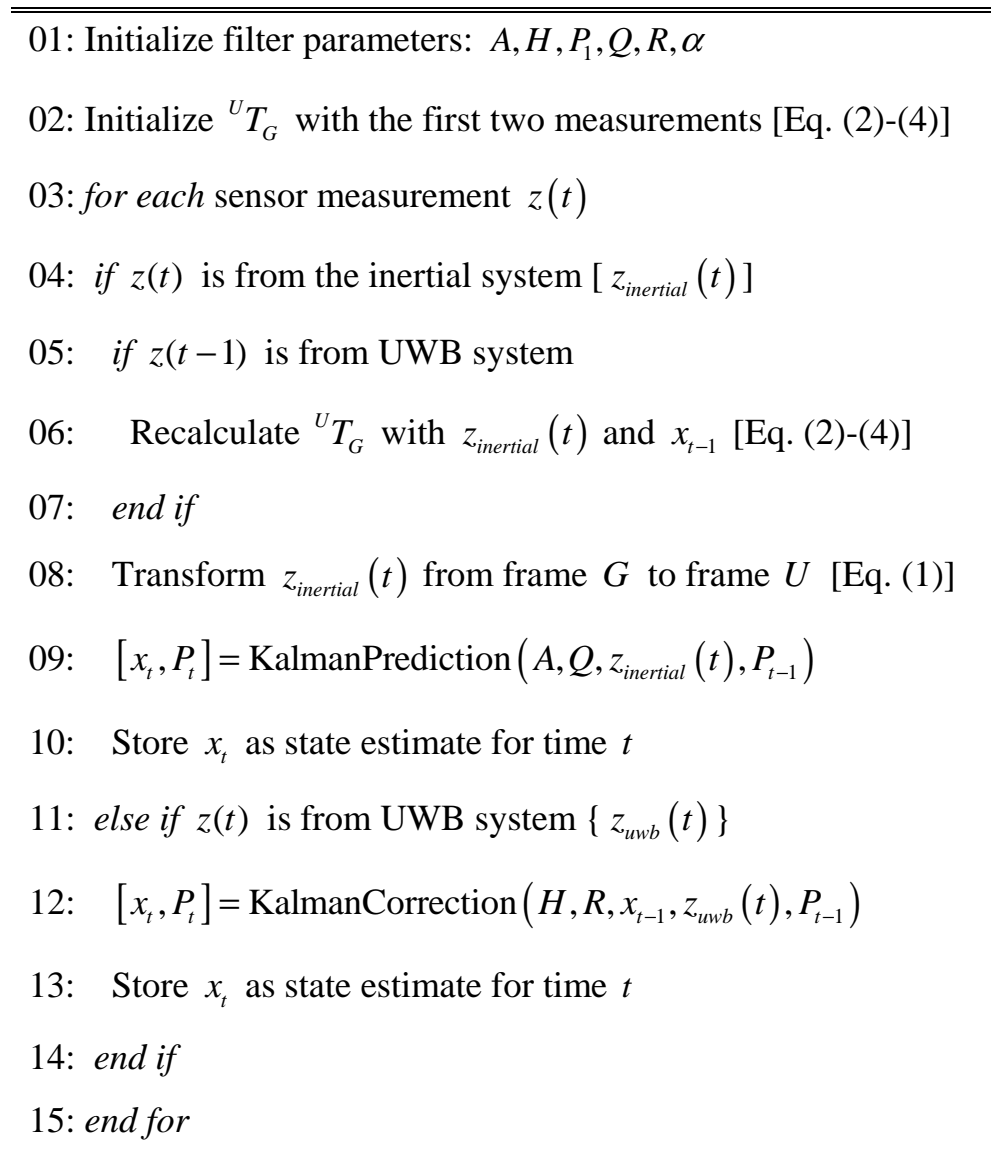

\subsection{Particle filter fusion approach}

Another widespread technique for implementing Bayesian filters is the particle filter. In this paper, a fusion algorithm derived from a particle filter algorithm with a Sampling Importance Resampling (SIR) scheme [17] has been developed in order to compare it with the Kalman filter approach.

For the application presented here, the prediction step of the SIR particle filter obtains a set $S_{t}$ of $n$ particles $x_{t}^{[i]}$ from the state transition distribution, which is modeled by adding a random Gaussian noise $w_{i}$ of zero mean and covariance matrix $Q$ to the position measurement from the motion capture system $Z_{\text {inertial }}(t)$ (see Equation 12). The covariance matrix $Q$ represents the error covariance of the motion capture system and its terms are computed as explained in the previous section. 


$$
S_{t}=\left\{x_{t}^{[i]}=z_{\text {inertial }}(t)+w_{i} \mid w_{i} \sim N(0, Q) ; i=1, \ldots, n\right\}
$$

The Gaussian error $w_{i}$ in (12) is obtained from the Cholesky decomposition $C$ of the covariance matrix $Q=C^{T} C$ and a normally distributed random vector $Z \sim N(0, I)$ :

$$
w_{i}=C^{T} \cdot Z \sim N\left(0, C^{T} \cdot C\right)=N(0, Q)
$$

In the importance factor calculation step, the weights $w_{t}^{[i]}$ of the particles are computed from the measurement model $p\left(z_{u w b}(t) \mid x_{t-1}^{[i]}\right)$. This probability is calculated as a multivariate normal distribution $N\left(z_{u w b}(t), R\right)$ with the UWB measurement $z_{u w b}(t)$ as mean and $R$ as covariance matrix. This normal distribution is evaluated for each particle $x_{t-1}^{[i]}$ of the prediction step (12). The covariance matrix $R$ represents the error covariance of the UWB system and its terms are computed as explained in the previous section. Finally, the weights of all the particles will be normalized to ensure that they sum up to one.

$$
\begin{aligned}
& w_{t}^{[i]} \sim N\left(z_{u w b}(t), R\right)=\frac{1}{(2 \pi)^{3 / 2} \operatorname{det}(R)^{1 / 2}} . \\
& \exp \left(-\frac{1}{2}\left(x_{t-1}^{[i]}-z_{u w b}(t)\right)^{T} R^{-1}\left(x_{t-1}^{[i]}-z_{u w b}(t)\right)\right)
\end{aligned}
$$

Sequential Importance Sampling (SIS) filters are only composed of these two steps. However, in many cases, after some iterations of these filters, only a few particles have non-zero importance weights. This problem is called weight degeneracy or sample impoverishment. The resampling step reduces this problem by defining a new particle set where state estimates with high weights are represented by several particles while state estimates with low weights are removed. The resampling algorithm draws with replacement $n$ particles from the initial particle set $S_{t}$. The probability of drawing each particle is given by its importance factor. In this paper the 'systematic resampling' algorithm presented in [18] has been used because it has a reduced computational complexity [19].

The implemented algorithm is described in detail in Table 2 . It has a very similar structure to the Kalman filter algorithm (Table 1): Motion capture measurements are used in the prediction step (line 9 of Table 2) and UWB measurements are used in the importance factor calculation step (line 12 of Table 2). The importance factor and resampling steps will only be executed with the frequency of UWB measurements. Thereby, the execution time of the algorithm is reduced with regard to previously developed particle filter algorithms ([13], [15]), which have different particle weighing channels for each sensor and have always to perform the weighing and resampling steps. Just after registering a UWB measurement, the transformation matrix ${ }^{U} T_{G}$ is recalculated (line 6 of Table 2) in order to correct the error accumulated by the previous measurements and remove it from the following state estimates. 
Table 2: Fusion algorithm based on Particle filter.

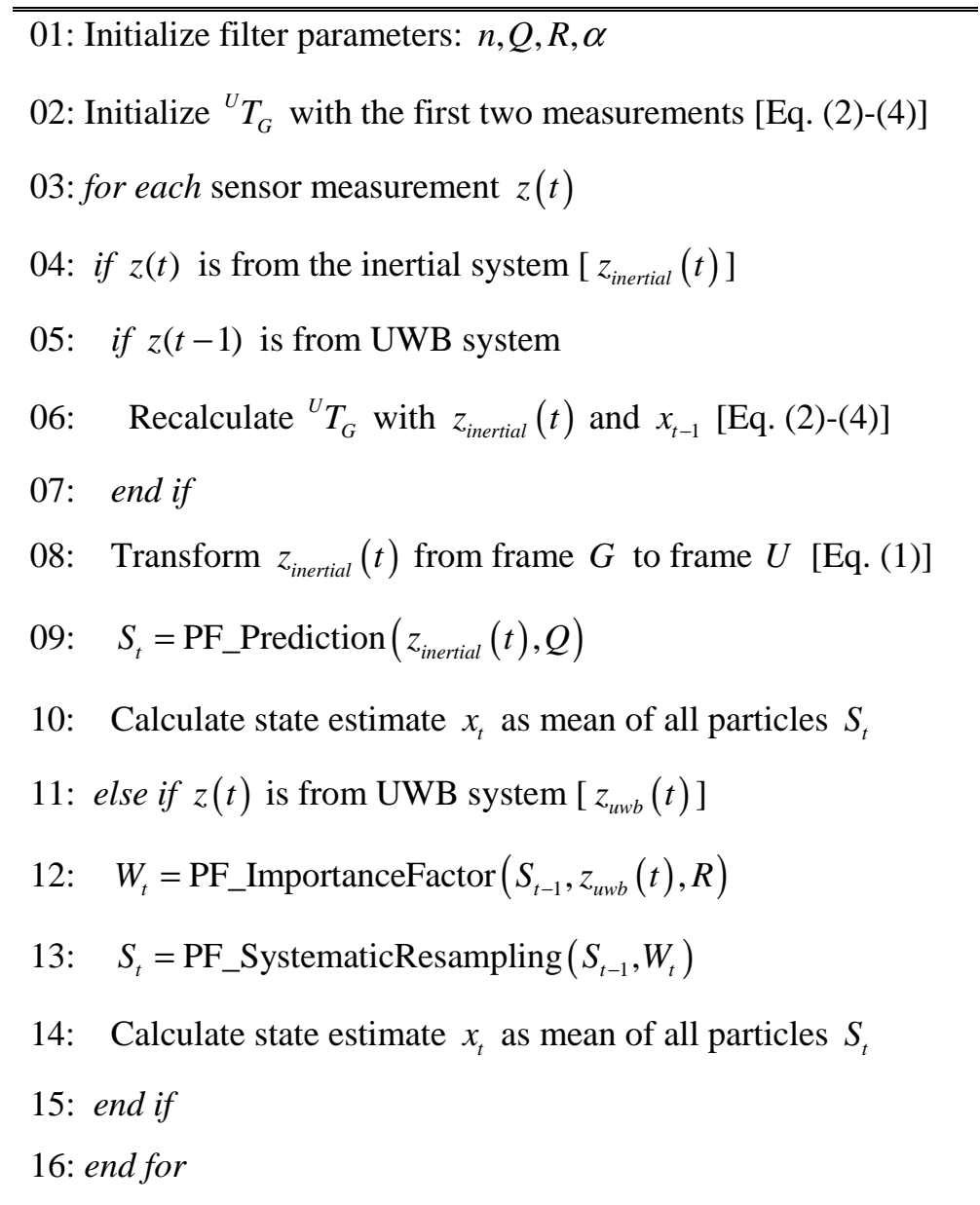

\subsection{Kalman-particle filter combination approach}

The third approach implemented in this paper relies on combining the previous two Bayesian techniques: Kalman filter and particle filter. The Kalman filter is applied to measurements from the inertial motion capture system while the SIR particle filter is applied to UWB measurements. Previous state estimates $\hat{x}_{t-1}$ are used in the prediction steps of these filters in order to interchange measurements between them and thus correct each other's errors.

The state evolution model of the prediction step in the Kalman filter is based on the previous state estimate $\hat{x}_{t-1}$ plus a Gaussian noise $w_{K F}(t)$ with zero mean and covariance $Q_{K F}$ (which represents the dispersion of the difference between the current position and the previous one):

$$
\hat{x}_{t}^{-}=\hat{x}_{t-1}+w_{K F}(t)
$$

This prediction step is implemented by the following equations (where $A$ is a $3 \times 3$ identity matrix):

$$
\begin{gathered}
\hat{x}_{t}^{-}=A \hat{x}_{t-1} \\
P_{t}^{-}=A P_{t-1} A^{T}+Q_{K F}
\end{gathered}
$$


The observation model of the correction step in the Kalman filter approximates the measurements of the inertial motion capture system from the state estimate of the prediction step $\hat{x}_{t}^{-}$plus a Gaussian noise $w_{\text {inertial }}(t)$ with zero mean and covariance $R_{K F}$ (which is equal to the covariance matrix $Q$ of the Kalman filter in section 4.3):

$$
\hat{z}_{\text {inertial }}(t)=\hat{x}_{t}^{-}+w_{\text {inertial }}(t)
$$

This observation model is implemented in the Kalman filter correction step using the following three equations (where $H$ is a $3 \times 3$ identity matrix and $z_{\text {inertial }}(t)$ is the motion capture system measurement):

$$
\begin{gathered}
K_{t}=P_{t}^{-} H^{T}\left(H P_{t}^{-} H^{T}+R_{K F}\right) \\
\hat{x}_{t}=\hat{x}_{t}^{-}+K_{t}\left(z_{\text {inertial }}(t)-H \hat{x}_{t}^{-}\right) \\
P_{t}=\left(I-K_{t} H\right) P_{t}^{-}
\end{gathered}
$$

Each time a UWB measurement is registered, the SIR particle filter is executed. In the prediction step of this particle filter, a set $S_{t}$ of $n$ particles $x_{t}^{[i]}$ is obtained by adding a random Gaussian noise $w_{P F}^{[i]}$ with zero mean and covariance $Q_{P F}$ to the state estimate $\hat{x}_{t-1}$ obtained with the Kalman filter from the previous inertial measurement:

$$
S_{t}=\left\{x_{t}^{[i]}=x_{t-1}+w_{P F}^{[i]} \mid w_{P F}^{[i]} \sim N\left(0, Q_{P F}\right) ; i=1, \ldots, n\right\}
$$

The importance factor calculation and resampling steps of this particle filter are implemented in a similar way to the previous particle filter algorithm described in section 4.4. The weights $w_{t}^{[i]}$ of the particles computed in the prediction step are obtained from a multivariate normal distribution $N\left(z_{u w b}(t), R_{P F}\right)$ while the resampling step is implemented by a systematic resampling approach [18]. The covariance matrix $R_{P F}$ contains the variance of the errors of the UWB system and it is equal to the $R$ covariance matrix of the particle filter in section 4.4.

The fusion algorithm obtained from the combination of this Kalman and particle filters is described in Table 3. This algorithm improves the performance of the particle filter algorithm presented in section 4.4 because its execution frequency is reduced. The high rate measurements from the motion capture systems are processed with the Kalman filter (lines 9-10 of Table 3) and thus the particle filter is only executed when slow measurements from the UWB system are obtained (lines 13-15 of Table 3). In a similar way to the fusion algorithms of sections 4.3 and 4.4, the transformation matrix ${ }^{U} T_{G}$ is recalculated (line 6 of Table 3) after an UWB measurement is received in order to correct the error accumulated by the inertial system, as in the previous fusion algorithms. 
Table 3: Fusion algorithm based on Kalman-particle filter.

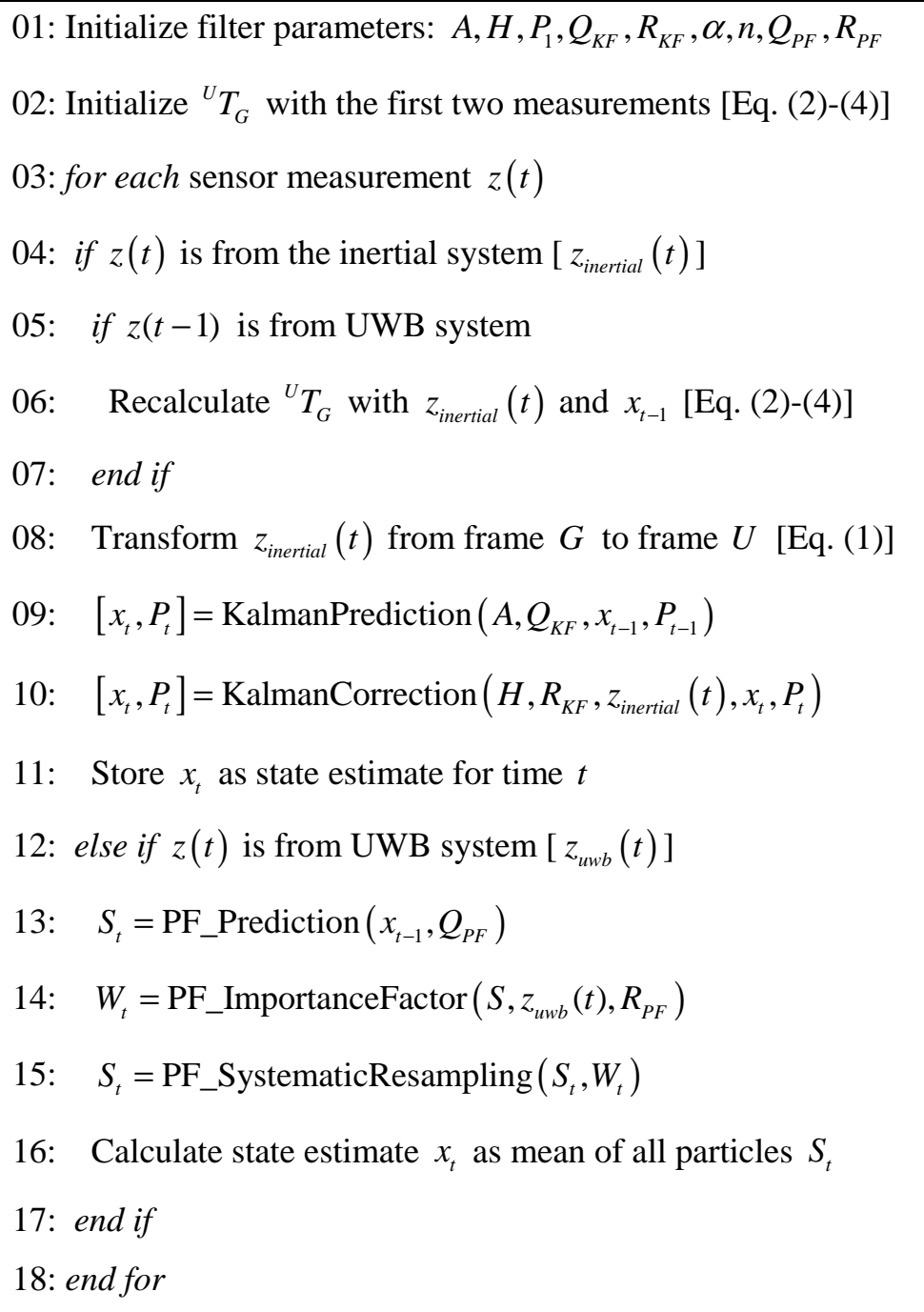

\section{EXPERIMENTAL RESULTS}

A collection of experiments has been developed in order to compare the precision and efficiency of the three Bayesian fusion filters described in section 4. Two paths have been defined in order to perform these experiments: a linear path of 3 meters and a rectangular path of 8 meters. Both trajectories are defined in the same XY plane with a constant height (coordinate $\mathrm{Z}$ ) since the errors in the vertical component are negligible in comparison with the errors in the horizontal plane. A human operator who wears the inertial motion capture suit and the UWB tag has covered each trajectory fifteen times at a walking speed of approximately $1-3 \mathrm{~m} / \mathrm{s}$. These trajectories are marked on the floor so that the human operator follows them with precision. The points of these marked trajectories are used as ground-truth values for computing the position errors of the tracking systems. The global translation measurements which are registered by the inertial motion capture system and the UWB system in these experiments are processed in three Matlab functions which implement the three Bayesian filter algorithms. All the algorithms have been tested in the same computer (Intel 
Pentium 4 2.8GHz processor, 760MB RAM) to compare their computational efficiency. A set of 100 particles has been used in the algorithms which are based on particle filtering.

The global position measurements obtained in one of the fifteen experiments of the linear path are depicted in Fig. 3. Fig. 3.a shows the original position measurements registered by the two tracking systems (inertial motion capture system and UWB system) in the same coordinate system. This plot shows the advantages and disadvantages of both tracking systems. On one hand, the inertial system obtains fast measurements which define a continuous movement while UWB measurements have a lower sampling rate. On the other hand, measurements from the inertial system accumulate an error which increases over time while UWB measurements maintain approximately a constant error. Therefore, the fusion algorithms developed in this paper should be applied to calculate better position estimates. Figs. 3.b, 3.c and 3.d depict the position estimates which are obtained in this experiment by applying the Kalman filter, the particle filter and the Kalman-particle filter fusion algorithms, respectively.

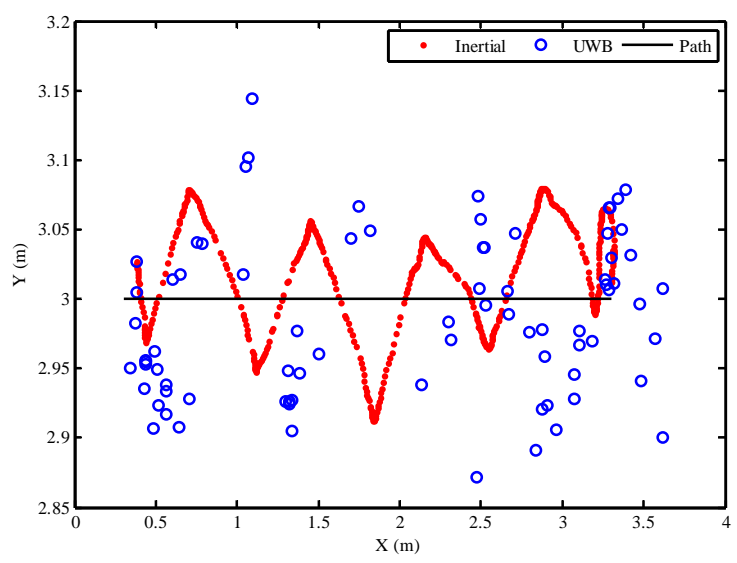

(a)

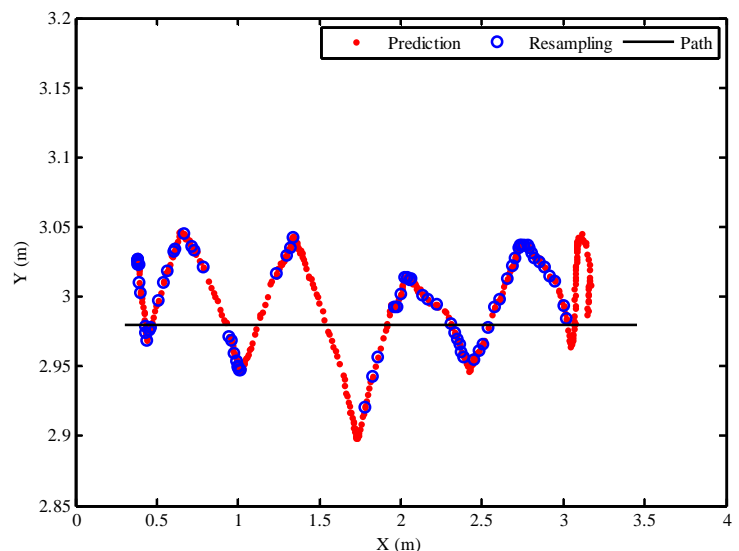

(c)

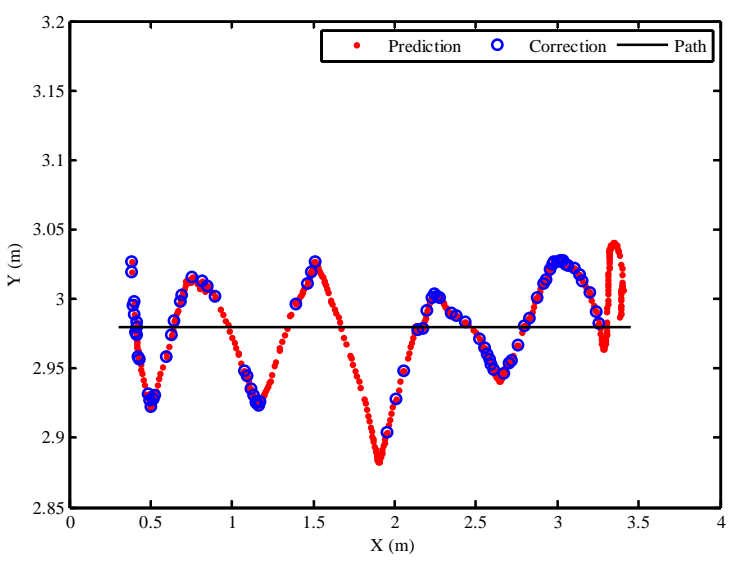

(b)

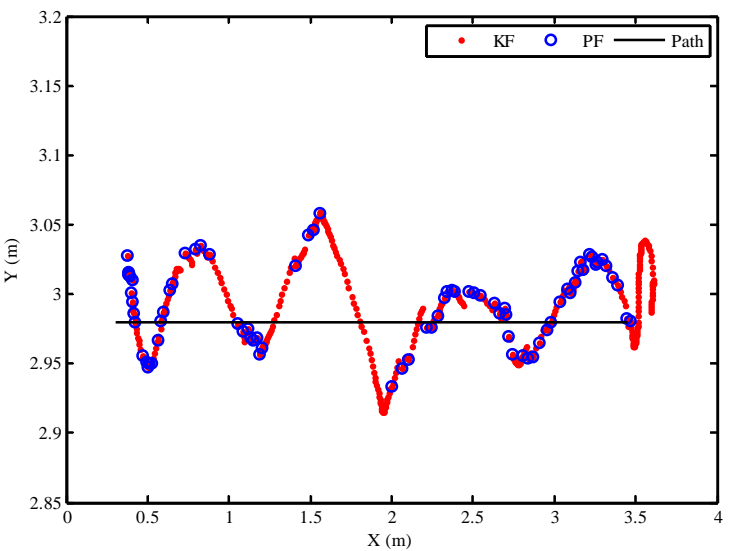

(d)

Figure 3: Linear trajectory experiment: (a) original position measurements from the inertial and UWB systems; (b) position estimates from the Kalman filter algorithm in the prediction and correction steps; (c) position estimates from the particle filter algorithm in the prediction and resampling steps; (d) position estimates from the Kalman-particle filter algorithm in the Kalman filter (KF) and the particle filter (PF) steps. 
Fig. 4 shows the error statistics (error mean and standard deviation) of all the position estimates computed by the three fusion algorithms along the fifteen experiments of the linear path. These statistics are obtained by calculating the Euclidean distance between each position measurement and the corresponding ground-truth point in the linear path. The Kalman-particle filter algorithm has a global mean error $(0.037 \pm 0.029 \mathrm{~m})$ very similar to the global error of the Kalman filter algorithm $(0.038 \pm 0.029 \mathrm{~m})$. The particle filter algorithm has a slightly higher global error $(0.044 \pm 0.029 \mathrm{~m})$.

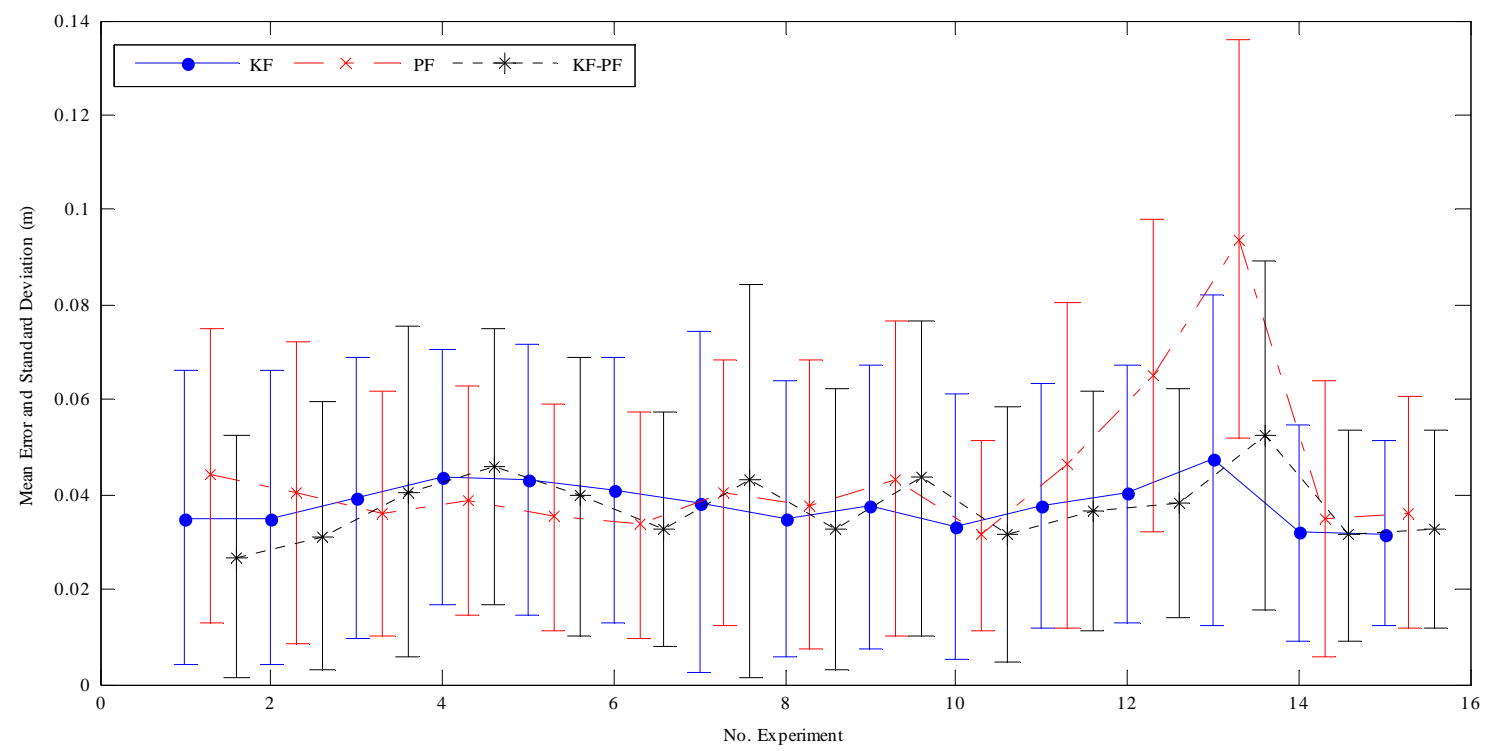

Figure 4: Mean error and standard deviation of the position estimates from the three fusion algorithms (KF: Kalman filter, PF: Particle filter, KF-PF: Kalman-particle filter) in the linear path experiments.

Fig. 5.a depicts the number of measurements of the inertial motion capture system and the number of measurements of the UWB localization system which are processed by the fusion algorithms at each experiment of the linear path. The number of measurements of the inertial system is always substantially higher than the number of measurements of the UWB system because of its higher sampling rate. Fig. 5.b shows the execution time which is spent by the fusion algorithms to combine all the measurements of both tracking systems. The Kalman filter is the least expensive algorithm in terms of execution time with a global mean time of $0.077 \mathrm{~s}$. The Kalman-particle filter algorithm has an intermediate computational cost (global mean execution time: 1.137s) between the Kalman filter and the particle filter (global mean execution time: 1.523s). 


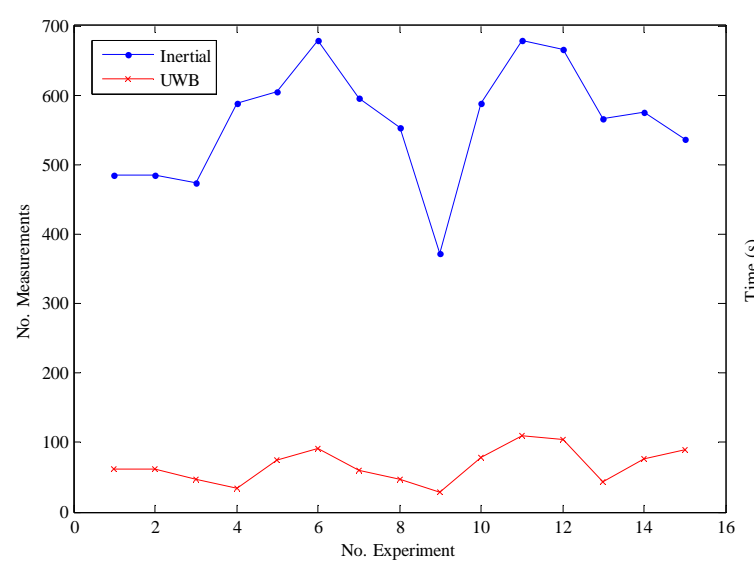

(a)

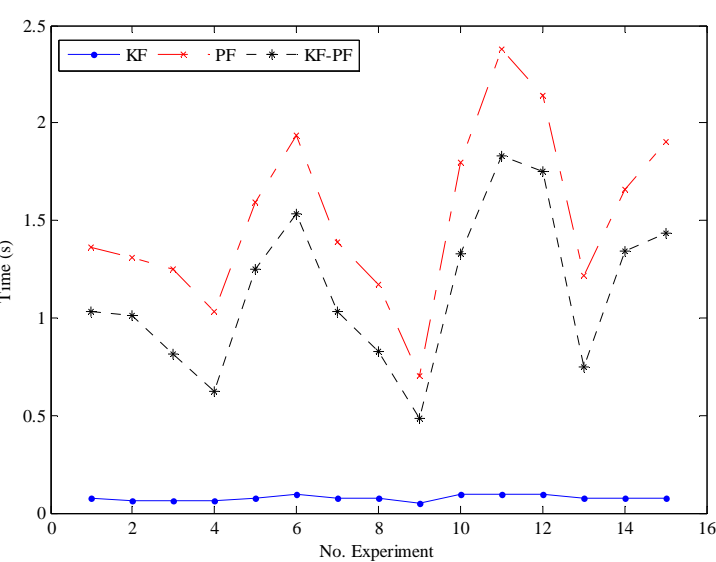

(b)

Figure 5: Computational cost of the linear path experiments: (a) number of measurements registered by the two tracking systems (inertial and UWB); (b) total execution time of the three fusion algorithms (KF: Kalman filter, PF: Particle filter, KF-PF: Kalman-particle filter).

Fig. 6.a depicts the original global position measurements registered by the tracking system during one of the fifteen experiments of the rectangular path. The error accumulation of the inertial system is more evident in this trajectory because it is longer than the previous linear path (Fig. 6.a). The differences in the accuracy of the position estimates obtained from the three fusion algorithms are also more important and they can be appreciated in the Figs. 6.b, 6.c and 6.d. The trajectory estimated by the Kalman-particle filter algorithm (Fig. 6.d) is the most precise because it has a tighter fit to the real rectangular trajectory. The trajectory estimated by the particle filter algorithm (Fig. 6.c) is the least precise because the estimation positions are farther from the real path.

A more in-depth description of the errors of the position estimates computed by the three fusion algorithms is shown in Fig. 7. As it was stated before, the Kalman-particle filter algorithm obtains more accurate position estimates (global mean error: $0.063 \pm 0.055 \mathrm{~m}$ ) than the Kalman filter (global mean error: $0.105 \pm 0.076 \mathrm{~m}$ ) and the particle filter algorithms (global mean error: $0.184 \pm 0.127 \mathrm{~m}$ ).

Fig. 8.a shows the number of measurements which are combined by the fusion algorithms in each experiment of the rectangular trajectory. In this case, the number of processed measurements is higher because the path is longer. Fig. 8.b depicts the execution time of the fusion algorithms to combine these measurements. On one hand, the number of measurements of the inertial system is the main factor that determines the different execution times of the same fusion algorithm at different experiments. On the other hand, when the execution times of the three fusion algorithms are compared, the same results than in the linear path experiments are obtained. The Kalman filter algorithm has a smaller computational cost (global mean execution time: 0.561s) than the Kalmanparticle filter (global mean execution time: 4.358s) and the particle filter algorithms (global mean execution time: $5.947 \mathrm{~s})$. 


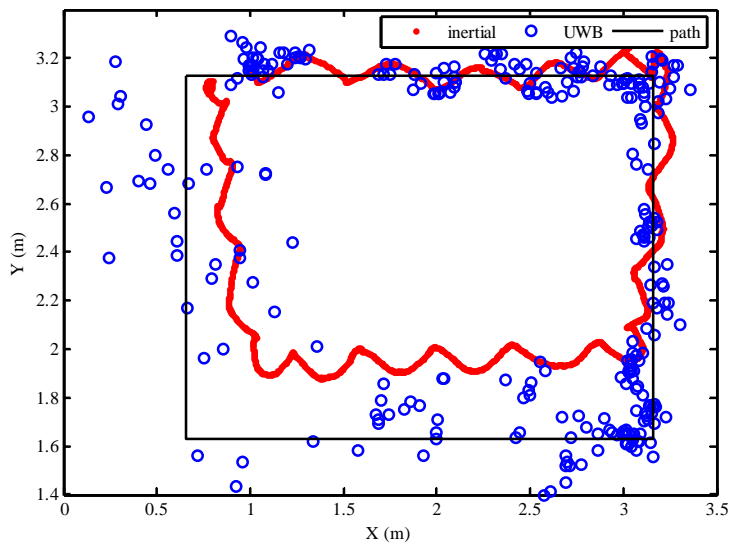

(a)

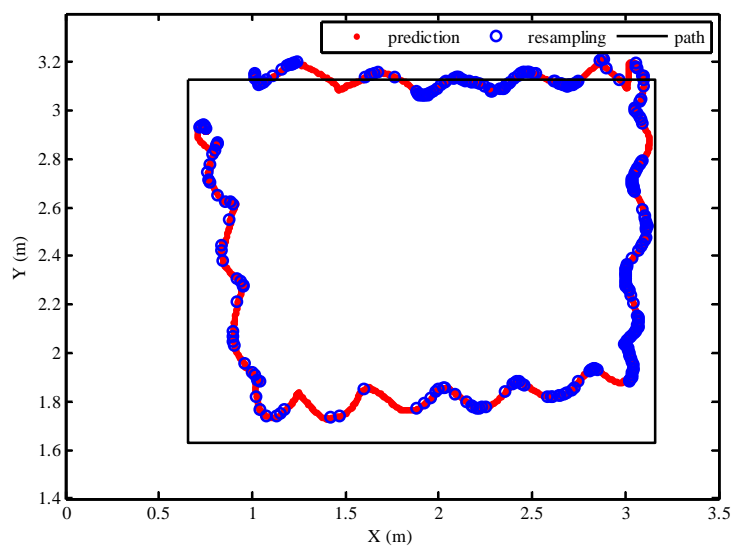

(c)

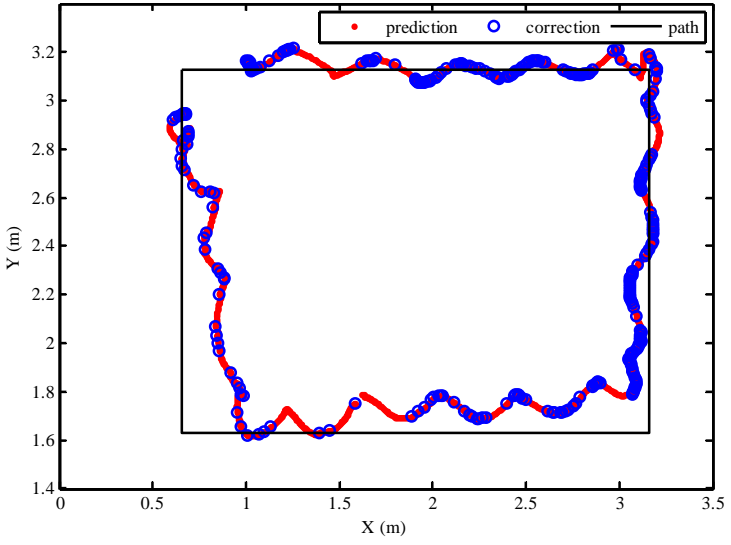

(b)

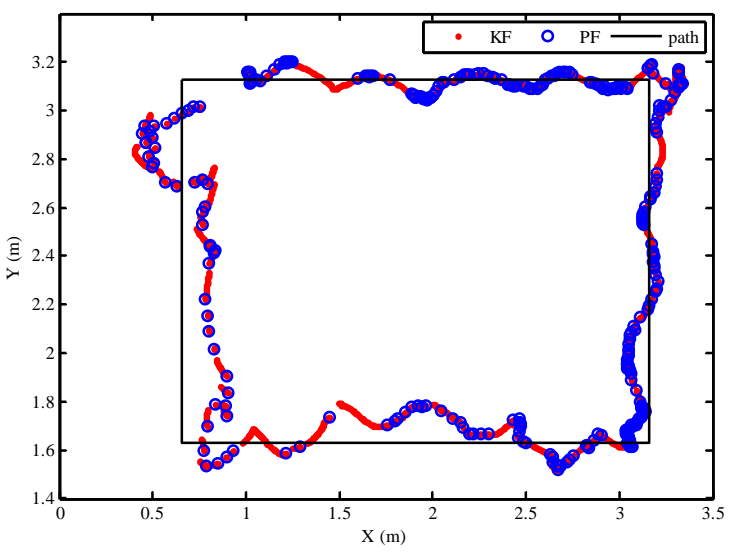

(d)

Figure 6: Rectangular trajectory experiment: (a) original position measurements from the inertial and UWB systems; (b) position estimates from the Kalman filter algorithm in the prediction and correction steps; (c) position estimates from the particle filter algorithm in the prediction and resampling steps; (d) position estimates from the Kalman-particle filter algorithm in the Kalman filter (KF) and the particle filter (PF) steps.

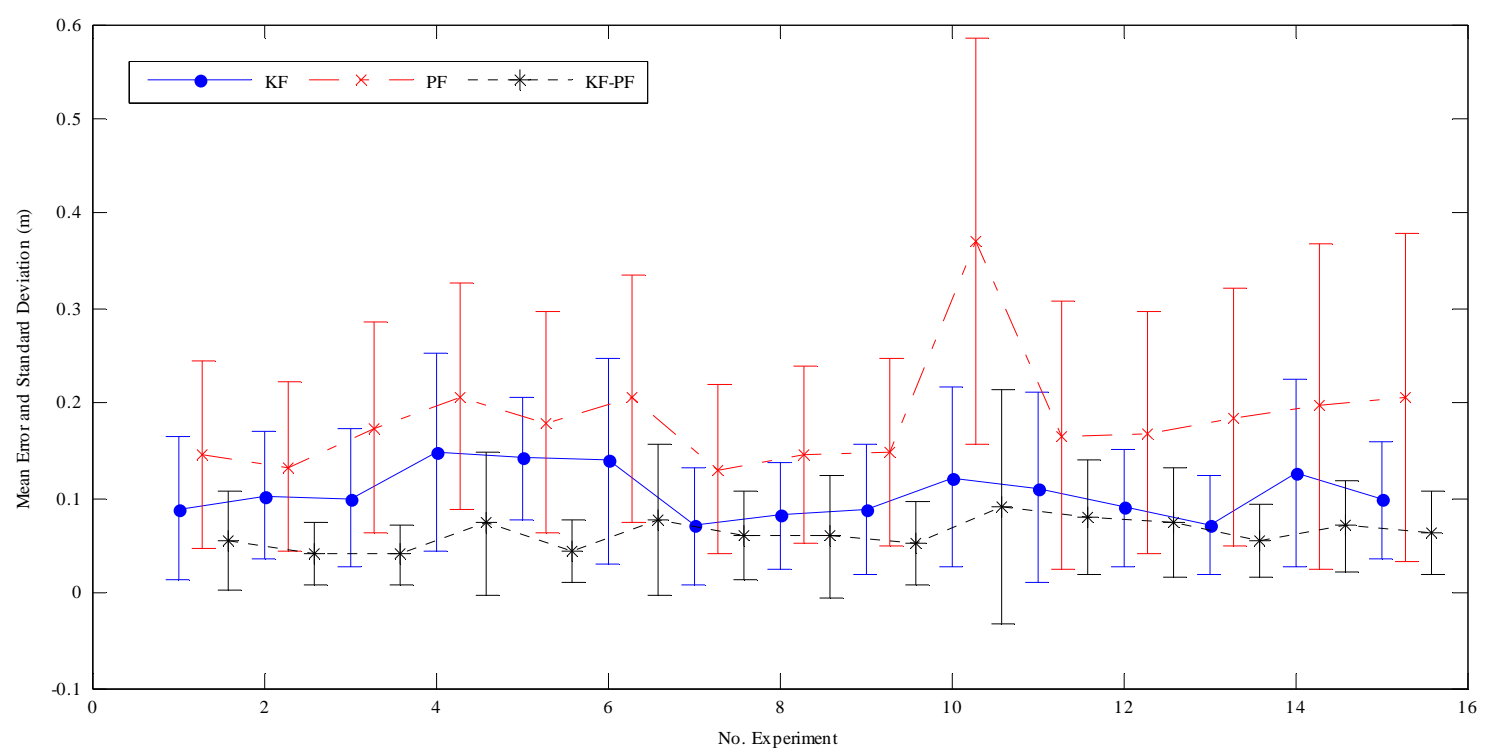


Figure 7: Mean error and standard deviation of the position estimates from the three fusion algorithms (KF: Kalman filter, PF: Particle filter, KF-PF: Kalman-particle filter) in the rectangular path experiments.

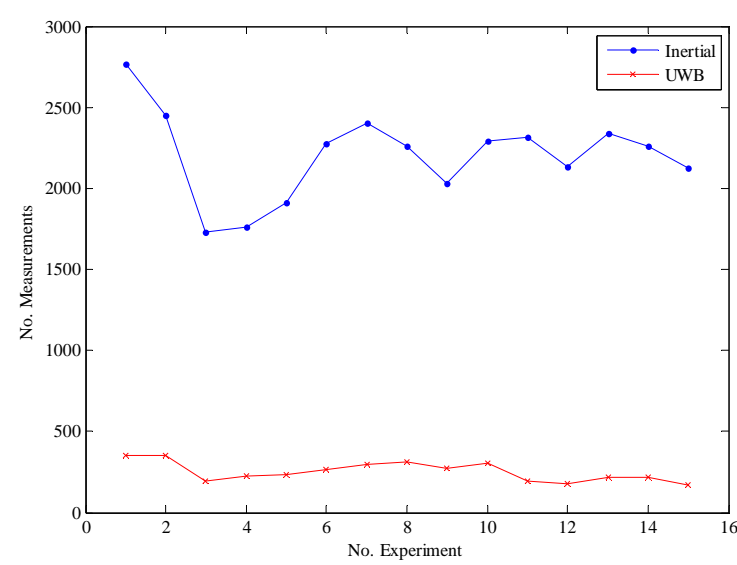

(a)

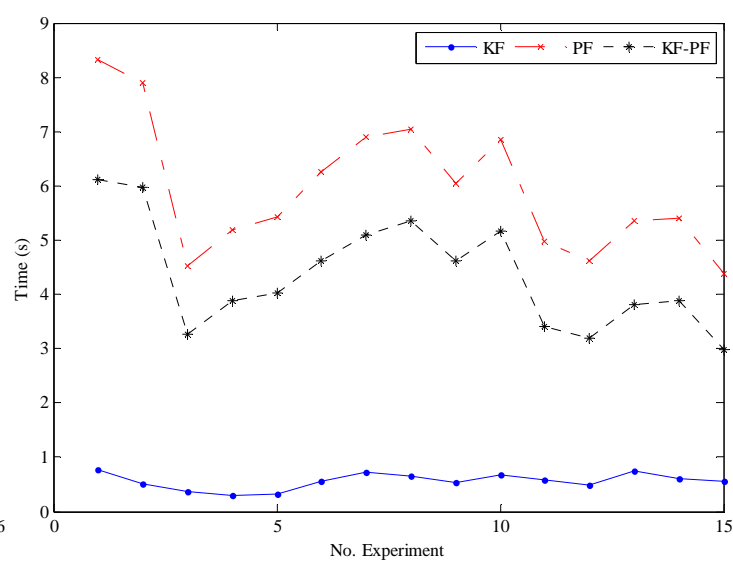

(b)

Figure 8: Computational cost of the rectangular path experiments: (a) number of measurements registered by the two tracking systems (Inertial and UWB); (b) total execution time of the three fusion algorithms (KF: Kalman filter, PF: Particle filter, KF-PF: Kalman-particle filter).

The three fusion algorithms developed in this research can be compared according to the results of the experiments described above. The Kalman-particle filter combination algorithm obtains the more precise position estimates. Therefore, this algorithm is suitable for human tracking in industrial environments. The better accuracy of this algorithm is based on its internal structure. The fast measurements of the inertial motion capture system can be easily represented with the linear Gaussian models of a Kalman filter while the slow measurements of the UWB system are better modeled with a nonlinear non-Gaussian technique (like a particle filter) because of their high latency. The fusion algorithm based on a Kalman filter is the most efficient fusion algorithm because of its simple implementation. However, it should be used in tracking systems where less accuracy is needed because it is not able to correct the errors of the inertial motion capture system as much as the Kalman-particle filter algorithm.

\section{CONCLUSIONS}

In this paper, three novel algorithms based on Bayesian Filtering have been developed to combine position measurements from two human tracking systems (an inertial motion capture system and a UWB localization system) with different sampling rates and accuracy. The first algorithm is based on a Kalman filter where measurements from the motion capture system are used in the prediction step of the filter and UWB measurements are incorporated in the correction step. The second algorithm is based on a particle filter and follows a general structure very similar to the Kalman 
filter: motion capture measurements are used in the prediction step and UWB measurements are used in the importance factor calculation step. Both algorithms use motion capture measurements in the motion model to predict the next position of the human operator. UWB measurements are used in the observation model to correct the previously predicted positions. The third algorithm combines these two Bayesian techniques: motion capture measurements are processed by a Kalman filter while UWB measurements are processed by a particle filter. In these three algorithms, the transformation matrix between the coordinate frames of both tracking systems is recalculated each time a UWB measurement is processed and thus next measurements from the motion capture system are corrected with the previous UWB position estimate.

These fusion algorithms have been compared by performing several experiments where a human operator has followed two different paths: a linear trajectory and a rectangular trajectory. Several conclusions can be drawn from the obtained results. Firstly, the fusion of both tracking systems has been able to correct the drift which made impractical the use of the inertial motion capture system for the global localization of a human operator. Secondly, the errors of position estimates obtained by the Kalman filter and the Kalman-particle filter approaches are assumable $(0.06-0.10 \mathrm{~m}$ in a $8 \mathrm{~m}$ trajectory) for the development of general human-robot interaction tasks. Thirdly, the development of several fusion algorithms enables the selection of one or the other solution depending on the efficiency and accuracy requirements of the application. Thereby, applications which require a quick response of the tracking system will use the Kalman filter algorithm while applications which require precise positioning will implement the Kalman-particle solution. Finally, the validity of the proposed algorithms depends on the supply of sufficient UWB measurements for the correction of the inertial drift. If the UWB system does not provide a minimum sampling rate, the inertial system will accumulate too much drift between each pair of UWB measurements and this error will make impossible the tracking of the human.

This paper has been focused on the precise localization of a human operator in an industrial environment. This is the first step to create intelligent industrial workplaces where human operators can interact naturally and unobtrusively with industrial machinery. Future work will be concentrated on developing real collaboration tasks between human operators and industrial robots where the tracking measurements obtained from these fusion algorithms will be used.

\section{ACKNOWLEDGMENTS}

This work is supported by the Spanish Ministry of Education and Science (MEC) under the research projects DPI2005-06222 and DPI2008-02647 and the grant AP2005-1458.

\section{REFERENCES}

[1] D. Kulic and E. Croft, Safe Motion Planning for Human-Robot Interaction: Design and Experiments, in: A. Lazinica, ed., Mobile Robots Towards New Applications, IN_TECH, Germany, 2006, pp. 149-170. 
[2] R. Alami, A. Albu-Schaeffer, A. Bicchi, R. Bischoff, R. Chatila, A.D. Luca, A.D. Santis, G. Giralt, J. Guiochet, G. Hirzinger, F. Ingrand, V. Lippiello, R. Mattone, D. Powell, S. Sen, B. Siciliano, G. Tonietti and L. Villani, Safe and Dependable Physical Human-Robot Interaction in Anthropic Domains: State of the Art and Challenges, in: Proc. IEEE/RSJ International Conference on Intelligent Robots and Systems, Workshop on Physical Human-Robot Interaction in Anthropic Domains, Beijing, 2006, pp. 1-15.

[3] G. Welch and E. Foxlin, Motion Tracking: No Silver Bullet but a Respectable Arsenal, IEEE Computer Graphics and Applications 22(6) (2002) 24-38.

[4] J.A. Corrales, F.A. Candelas and F. Torres, Hybrid tracking of human operators using IMU/UWB data fusion by a Kalman filter, in: Proc. ACM/IEEE International Conference on Human-Robot Interaction, Amsterdam, The Netherlands, 2008, pp. 193-200.

[5] N. El-Sheimy, H. Hou and X. Niu, Analysis and Modeling of Inertial Sensors Using Allan Variance, IEEE Transactions on Instrumentation and Measurement 57(1) (2008) 140-149.

[6] V. Fox, J. Hightower, L. Lin, D. Schulz and G. Borriello, Bayesian Filtering for Location Estimation, IEEE Pervasive Computing 2(3) (2003) 24-33.

[7] D. Simon, Kalman Filtering, Embedded Systems Programming 14(6) (2001) 72-79.

[8] E. Foxlin, Inertial Head-Tracker Sensor Fusion by a Complementary Separate-Bias Kalman Filter, in: Proc. IEEE Virtual Reality Annual International Symposium, Santa Clara, California, 1996, pp. 185-194.

[9] E. Foxlin, Pedestrian Tracking with Shoe-Mounted Inertial Sensors, IEEE Computer Graphics and Applications 25(6) (2005) 38-46.

[10] D. Roetenberg, P.J. Slycke and P.H. Veltink, Ambulatory Position and Orientation Tracking Fusing Magnetic and Inertial Sensing, IEEE Transactions on Biomedical Engineering 54(5) (2007) 883-890.

[11] S. You and U. Neumann, Fusion of Vision and Gyro Tracking for Robust Augmented Reality Registration, in: Proc. IEEE Virtual Reality 2001 Conference, Yokohama, Japan, 2001, pp. 71-78.

[12] F. Caron, E. Duflos, D. Pomorski and P. Vanheeghe, GPS/IMU Data Fusion Using Multisensor Kalman Filtering: Introduction of Contextual Aspects, Information Fusion 7(2) (2006) 221-230.

[13] F.E. Ababsa and M. Mallem, Hybrid Three-Dimensional Camera Pose Estimation Using Particle Filter Sensor Fusion, Advanced Robotics 21(1) (2007) 165-181.

[14] D.B. Jourdan, J.J. Deyst, Jr., M.Z. Win and N. Roy, Monte Carlo localization in dense multipath environments using UWB ranging, in: Proc. IEEE International Conference on Ultra-Wideband (ICU 2005), Zurich, Switzerland, 2005, pp. 314-319. 
[15] J. Hightower and G. Borriello, Particle Filters for Location Estimation in Ubiquitous Computing: A Case Study, in: Proc. Sixth International Conference on Ubiquitous Computing (Ubicomp 2004), Nottingham, England, 2004, pp. 88-106.

[16] D. Roetenberg, H. Luinge and P. Slycke, 6 DOF Motion Analysis Using Inertial Sensors, in: Proc. Measuring Behavior Conference, Maastricht, The Netherlands, 2008, pp. 14-15.

[17] M.S. Arulampalam, S. Maskell, N. Gordon and T. Clapp, A Tutorial on Particle Filters for Online Nonlinear/Non-Gaussian Bayesian Tracking, IEEE Transactions on Signal Processing 50(2) (2002) 174188.

[18] J. Carpenter, P. Clifford and P. Fearnhead, Improved Particle Filter for Nonlinear Problems, IEE Proceedings Radar, Sonar and Navigation 146(1) (1999) 2-7.

[19] J.D. Hol, T.B. Schon and F. Gustafsson, On Resampling Algorithms for Particle Filters, in: Proc. Nonlinear Statistical Signal Processing Workshop, Cambridge, United Kingdom, 2006. 